\title{
Análise da política editorial de periódicos da ciência da informação: Biblionline e Encontros Bibli ${ }^{1}$
}

\author{
Edna da Silva Angelo \\ ednasangelo@gmail.com \\ Fabiene Letizia Alves Furtado \\ fabiene.furtado@gmail.com \\ Gracilene Maria Carvalho \\ gracilenecarvalho03@gmail.com \\ Nivaldo Calixto Ribeiro \\ zoopas@gmail.com
}

Resumo: Divulgar novos conhecimentos é relevantes para área de atuação deve ser a prioridade de um periódico científico. Para isso, faz-se necessária uma política editorial bem estruturada que garanta a sua qualidade e sustentabilidade. Neste contexto, objetivou-se com esse estudo analisar a política editorial dos periódicos da área de Ciência da Informação: Encontros Bibli e Biblionline e averiguar similaridades e diferenças de diretrizes de trabalho propostas por esses títulos e se influenciam no impacto de suas publicações e sua classificação em ferramentas de avaliação. Trata-se de uma pesquisa descritiva pelo fato de propor a descrição das características dos periódicos e estabelecer relações entre variáveis. Do ponto de vista dos procedimentos técnicos é uma pesquisa documental, uma vez que utiliza documentos já existentes e que não receberam tratamento analítico. $O$ viés da análise é qualitativo, já que levanta dados que expõem a qualidade dos periódicos em análise. Para a coleta dos dados, as principais fontes foram o web site dos periódicos Biblionline e Encontros Bibli. Além disso, foi construído um instrumento com 10 critérios relacionados a editoração de revistas científicas. Como insumos tecnológicos utilizou de uma planilha colaborativa, um documento compartilhado no Google Drive e o Ucinet, que foi utilizado para a elaboração de gráficos. Considerou-se que a endogenia e indexadores são indícios de possíveis de impactos para os indicadores dos periódicos em análise. Conclui-se que uma política editorial robusta, transparente e bem estruturada é fundamental para o reconhecimento do periódico.

Palavras-chave: Periódicos Científicos. Políticas editoriais. Indicadores de qualidade.

\footnotetext{
${ }^{1}$ Estudo desenvolvido na disciplina Comunicação Científica e Periódico Eletrônico do Programa de Pós-Graduação em Gestão e Organização do Conhecimento da Universidade Federal de Minas Gerais (PPG-GOC/UFMG). Dúvidas, esclarecimentos, críticas ou sugestões de melhorias podem ser encaminhadas diretamente para o e-mail dos autores ou realizadas no documento editável, disponível em: https://docs.google.com/document/d/1Sil8C553Fj-sMQvliZ6I6zw7KId2CPNIYAFg657ewHk/edit, até dia 30 de abril de 2021.
} 


\section{INTRODUÇÃO}

Os periódicos científicos, ou revistas científicas, têm como missão difundir o avanço científico no âmbito nacional e internacional, uma vez que representam o principal canal formal de disseminação da ciência e de legitimação da autoria das descobertas científicas (RIBEIRO, 2006).

$\mathrm{Na}$ atualidade, no Brasil, o mecanismo mais requisitado de classificação para periódicos é o WebQualis, ou simplesmente Qualis (CONTREIRA; MIRANDA, 2020). Esse instrumento foi criado exclusivamente para avaliar a produção científica dos programas de pós-graduação stricto sensu, agregando o aspecto quantitativo ao qualitativo (BARATA, 2016). Atenta-se que embora analise a produção bibliográfica, classificar revistas científicas nunca fez parte de seus objetivos. Mesmo assim, é reconhecido como método de avaliação por vários editores, autores e pesquisadores (CONTREIRA; MIRANDA, 2020).

Observando essa demanda de qualificação e visibilidade necessária das revistas científicas, pode-se afirmar que as políticas editoriais assumem um caráter estratégico essencial no seu desempenho e no seu reconhecimento, em função das suas características multidisciplinares. Nesse contexto, esta pesquisa buscou analisar dois periódicos científicos da área da Ciência da Informação com distintos indicadores. Enquanto o Biblionline não possui Fator de Impacto e a classificação Qualis B5, para o quadriênio 2013-2016, o Encontros Bibli destaca-se no cenário internacional e nacional tem Qualis $\mathrm{A} 2$, ambos para a área de comunicação e informação. Assim, o objetivo desta análise centra-se na averiguação de similaridades e de diferenças nas políticas editoriais e se influenciam nesse cenário.

Esta análise faz parte das atividades propostas na Disciplina Comunicação Científica e Periódico Eletrônico, ocorrida no primeiro semestre de 2020, pelo Programa de Pós-Graduação em Gestão \& Organização do Conhecimento da Universidade Federal de Minas Gerais, ministradas pelas Professoras Doutoras Célia da Consolação Dias e Dalgiza Andrade Oliveira.

É importante confrontar os resultados pois assim é possível identificar o que mais os periódicos devem levar em consideração para alcançar melhores qualificações. Acredita-se que se deve fomentar esse veículo sendo a informação 
um bem público e por esse viés democratizar com excelência o acesso ao conhecimento produzido.

\section{BREVE HISTÓRICO DO PERIÓDICO BIBLIONLINE}

A Biblionline (ISSN 1809-4775) é um periódico eletrônico de acesso aberto voltado para as áreas de Biblioteconomia, Arquivologia, Ciência da Informação e Museologia. Vinculado ao Departamento de Ciência da Informação da Universidade Federal da Paraíba (Brasil), vem sendo publicada ininterruptamente desde 2005 (BIBLIONLINE, 2020). A figura 1 apresenta sua página inicial.

Figura 1 - Página inicial periódico Biblionline

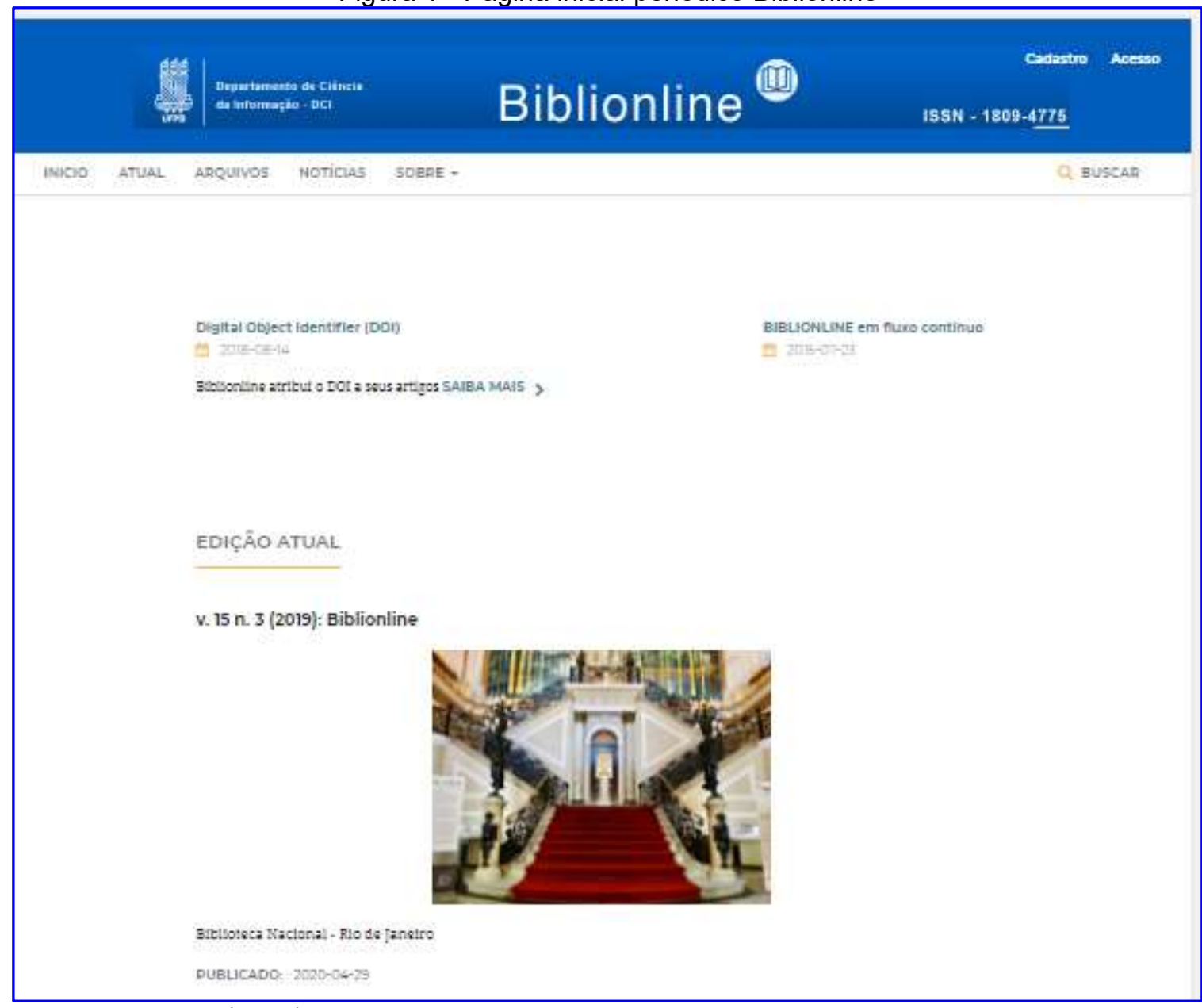

Fonte: Biblionline (2020)

Inicialmente tinha periodicidade semestral, entretanto devido à grande demanda, passou a ter periodicidade trimestral a partir de 2017. É publicado nos meses de abril, agosto e dezembro e recebe submissões em fluxo contínuo (BIBLIONLINE, 2020). Está classificado pelo Qualis quadriênio 2013-2016 nas 
seguintes áreas, conforme figura 2:

Figura 2 - Qualis quadriênio 2013-2016 periódico Biblionline

\begin{tabular}{|c|c|c|c|}
\hline \multicolumn{4}{|l|}{ Periódicos } \\
\hline ISSN & Titulo & Área de Avaliaçăo & Classificaçäo \\
\hline $1809-4775$ & BIBLIONLINE (JOÃO PESSOA) & ADMINISTRAC̣ÃO PÚBLLCA E DE EMPRESAS, CIÉNCIAS CONTÁBEIS E TURISMO & 84 \\
\hline $1809-4775$ & BIBLIONLINE (JOÃO PESSOA) & CIÊNCIAS AMBIENTAIS & 83 \\
\hline $1809-4775$ & BIBLIONLINE (JOÃO PESSOA) & COMUNICAÇÃO E INFORMAC̣ÃO & B5 \\
\hline $1809-4775$ & BIBLIONLINE (JOÃO PESSOA) & EDUCAÇÃO & B5 \\
\hline $1809-4775$ & BIBLIONLINE (JOÃO PESSOA) & ENGENHARIAS III & c \\
\hline $1809-4775$ & BIBLIONLINE (JOÃO PESSOA) & HISTÓRIA & B5 \\
\hline $1809-4775$ & BIBLIONLINE (JOÃO PESSOA) & INTERDISCIPLINAR & B2 \\
\hline $1809-4775$ & BIBLIONLINE (JOÃO PESSOA) & LINGUISTICA E LITERATURA & c \\
\hline $1809-4775$ & BIBLIONLINE (JOÃO PESSOA) & MEDICINA VETERINÁRIA & B5 \\
\hline $1809-4775$ & BIBLIONLINE (JOÃO PESSOA) & SERVIÇO SOCIAL & Bз \\
\hline
\end{tabular}

Fonte: Coordenação de Aperfeiçoamento de Pessoal de Nível Superior - Capes (2020).

Recentemente, Capes propôs um novo sistema de avaliação, cuja estratificação indicada é: $A 1, A 2, A 3, A 4, B 1, B 2, B 3, B 4$ e $C$, para periódicos não científicos. Entre as modificações, os periódicos passaram a ser avaliados em apenas uma área. Nessa nova classificação a Biblionline passou do estrato B5 para B2.

\section{BREVE HISTÓRICO DO PERIÓDICO ENCONTROS BIBLI}

Encontros Bibli: revista eletrônica de biblioteconomia e ciência da informação (ISSN 1518-2924) é uma publicação periódica do Programa de Pós-graduação em Ciência da Informação da Universidade Federal de Santa Catarina (Brasil) que tem como missão contribuir para a disseminação e promoção de novos conhecimentos em Ciência da Informação, Biblioteconomia, Arquivologia, Museologia e áreas correlatas (ENCONTROS BIBLI, 2020). A figura 3 apresenta sua página inicial. 
Figura 3 - Página inicial periódico Encontros Bibli

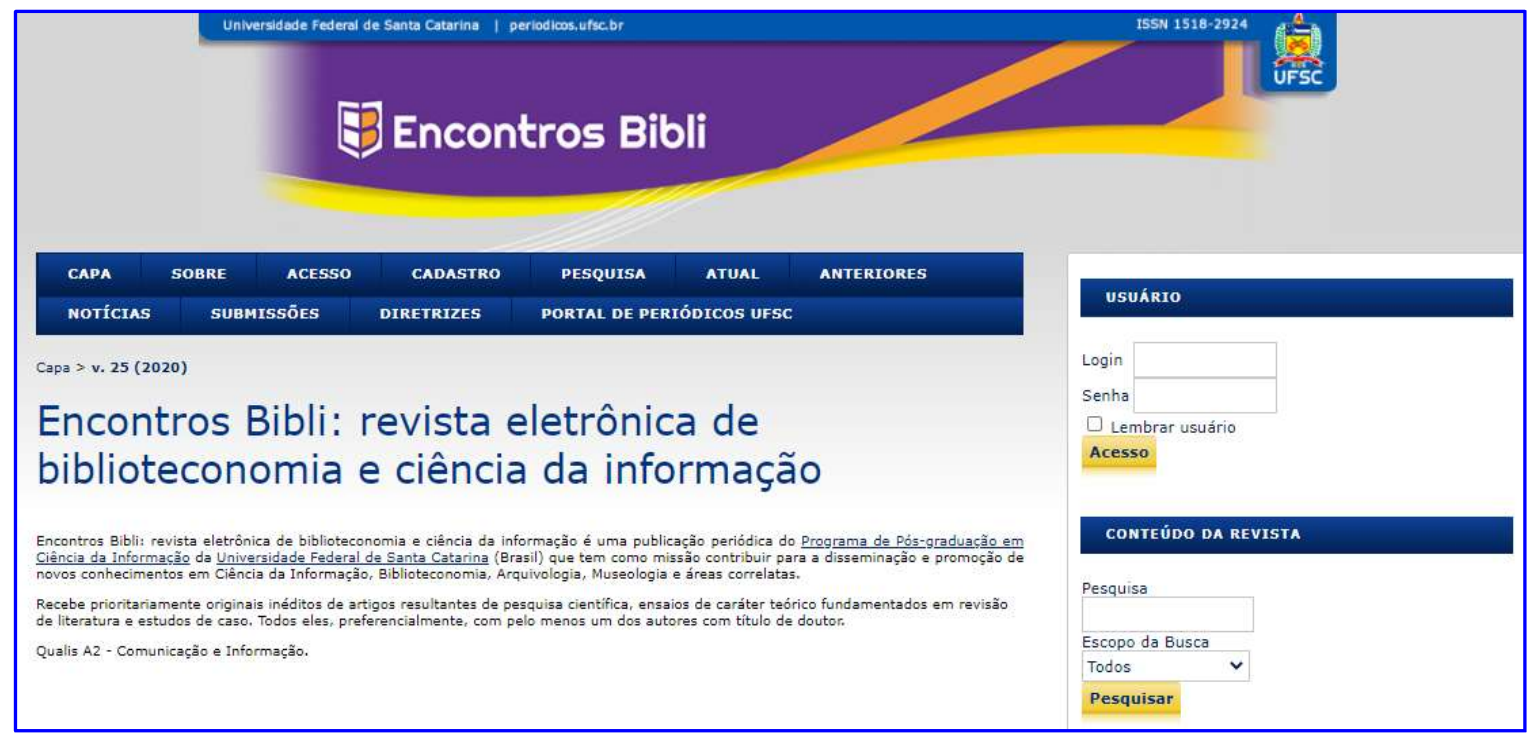

Fonte: Encontros Bibli (2020).

Foi criado em 1996. A partir de 2012 passa a ter periodicidade quadrimestral e em março de 2019 adota a publicação contínua. A partir de novembro de 2019 forma parte da Web of Science (ENCONTRO BIBLI, 2020). Esse é reconhecido como um dos mais importantes indexadores de conteúdo científico do mundo (PINTO; ANDRADE, 1999).

Na nova classificação proposta pela Capes a Encontros Bibli permaneceu no estrato A2 para área de Comunicação e Informação. Está classificado pelo Qualis quadriênio 2013-2016 nas demais áreas, conforme figura 4:

Figura 4 - Qualis quadriênio 2013-2016 periódico Encontros Bibli 


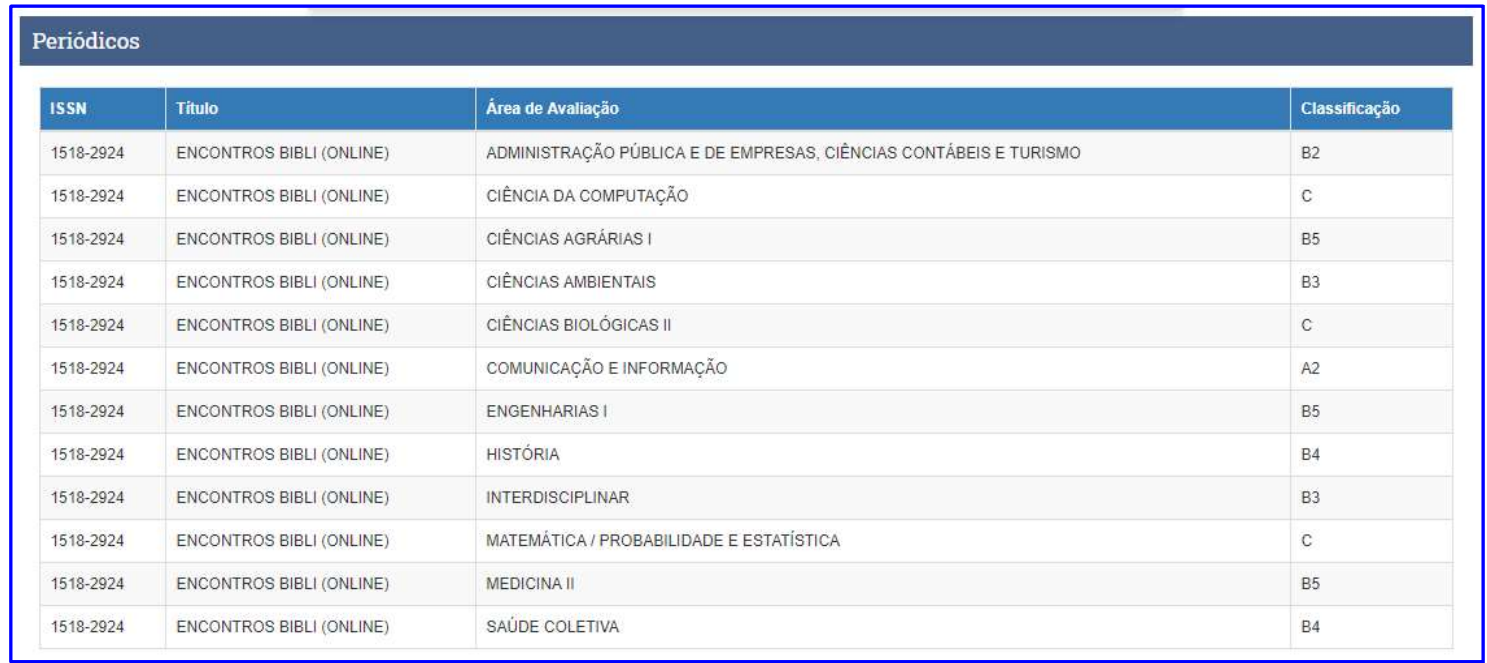

Fonte: Coordenação de Aperfeiçoamento de Pessoal de Nível Superior - Capes (2020).

Em 2012, foi lançado o Google Scholar Metrics com o objetivo de medir o impacto das publicações indexadas no Google Scholar. Essa ferramenta vem sendo especulada como possível alternativa ao Journal Citations Report (JCR) e ao SJR para avaliação da produção científica (ZHANG; LUN, 2019). De acordo com Costa, Canto e Pinto (2020) indexação adotada nessa ferramenta é automatizada e até o momento o periódico ter pelo menos 100 trabalhos publicados no período de avaliação e ao menos uma citação são os únicos critérios de inclusão na análise. Nessa classificação a Encontros Bibli foi classificada com H5 9 e o Mediano 12.

De acordo com o Google Metrics Scholar (2020) o índice h5 é o índice h dos artigos publicados nos últimos 5 anos completos e o $\mathrm{H}$ mediano é o número médio de citações dos artigos que compõem seu índice h5.

Um marca da Encontro Bibli está relacionado com a transparência e com abertura da ciência. Recentemente, a política da revista passou a permitir a submissão de artigo de dados. Na modalidade de Artigo de Dados, o conjunto de dados encaminhados ou disponibilizados podem ser: dados brutos, dados primários ou não processados, que estejam sendo descritos através de tabelas, gráficos ou figuras no artigo.

Uma inovação é que, devidamente autorizado, o parecer dos avaliadores sobre alguns dos artigo publicados tem sido publicitados. Outra, trata-se de uma regramento interessante, relacionado à submissão de trabalhos que refere-se ao documento intitulado "Notas da obra". Além das informações do artigo, consta licenças de usos, informações sobre o conjunto de dados, sobre os papéis que descrevem a contribuição específica de cada colaborador para a produção acadêmica: concepção e elaboração do manuscrito, coleta de dados, análise de 
dados, discussão dos resultados, revisão e aprovação e outros que se fizerem necessário.

\section{TRAÇOS METODOLÓGICOS}

De acordo com o objetivo desta pesquisa, é caracterizada como descritiva pelo fato de propor a descrição das características dos periódicos e estabelecer relações entre variáveis. Neste tipo de pesquisa não há interferência. Os fatos são observados, registrados, analisados, classificados e interpretados, sem que o pesquisador intervenha neles (BARROS; LEHFELD, 2007).

Do ponto de vista dos procedimentos técnicos é uma pesquisa documental, uma vez que utiliza documentos já existentes e que não receberam tratamento analítico (GIL, 1994). Para Prodanov e Freitas (2013, p. 54), esse tipo de pesquisa é destacada no momento em que pode-se "organizar informações que se encontram dispersas, conferindo-lhe uma nova importância como fonte de consulta".

Com relação ao viés da análise, entende-se que pode ser considerado como um estudo qualitativo, já que levanta dados que expõem qualidade dos periódicos. Para Godoy (1995), a pesquisa qualitativa tem o ambiente natural como fonte direta de dados e o pesquisador como instrumento fundamental.

Para a coleta dos dados, as principais fontes utilizadas foram o web site dos periódicos Biblionline e Encontros Bibli. As informações foram extraídas no mês de agosto de 2020. No intuito auxiliar a análise dos dados, foi criado um instrumento abordando 10 critérios, listadas a seguir:

a) padrão de normalização;

b) dados abertos;

c) equipe editorial;

d) nível de inserção do periódico no cenário internacional;

e) tempo de avaliação;

f) rede colaboração da revista;

g) cobertura temática do artigo;

h) diretrizes para autores indexadores da revista;

i) arquitetura da informação adotada;

j) Digital Object Identifier (DOI). 
Como procedimento de estruturação dos dados, utilizou-se uma planilha colaborativa do Google. Para a construção e visualização do grafo foi utilizado o programa Ucinet, programa especializado na análise sociométrica.

\section{RESULTADOS}

Nesta seção é apresentado o instrumento desenvolvido para a análise das políticas editoriais dos periódicos selecionados.

\begin{tabular}{|c|c|c|}
\hline \multicolumn{3}{|c|}{ Análise da política editorial de periódico da Ciência da Informação } \\
\hline Identificação & ISSN & Link de acesso \\
\hline Biblionline & $1809-4775$ & $\begin{array}{l}\text { https://periodicos.ufpb.br/ojs2/index.php/biblio/in } \\
\text { dex }\end{array}$ \\
\hline Encontros Bibli & $1518-2924$ & https://periodicos.ufsc.br/index.php/eb/index \\
\hline \multicolumn{3}{|c|}{ Padrão de normalização } \\
\hline Biblionline & $\begin{array}{l}\text { Associação } \\
6022 \text {-- Apr } \\
\text { NBR } 6023 \\
\text { Numeração } \\
6028 \text {-- Res } \\
\text { documentos } \\
\text { bibliográfico } \\
\text { Sobre a Rev }\end{array}$ & $\begin{array}{l}\text { Brasileira de Normas Técnicas (ABNT): NBR } \\
\text { esentação de artigos de publicações periódicas } \\
\text {-- Referências bibliográficas NBR } 6024 \text {-- } \\
\text { progressiva das seções de um documento NBR } \\
\text { umos NBR } 10520 \text {-- Apresentação de citações em } \\
\text { O texto segue os padrões de estilo e requisitos } \\
\text { s descritos em Diretrizes para Autores, na seção } \\
\text { ista. }\end{array}$ \\
\hline Encontros Bibli & $\begin{array}{l}\text { Citações e } \\
\text { normas da } \\
\text { ABNT. As } \\
\text { autor/data c }\end{array}$ & $\begin{array}{l}\text { referências: devem ser feitas com base nas } \\
\text { Associação Brasileira de Normas Técnicas - } \\
\text { citações devem ser feitas utilizando o sistema } \\
\text { om base na NBR } 10520 / 2002 \text { e as referências }\end{array}$ \\
\hline
\end{tabular}


devem utilizar a NBR 6023/2018.

\section{Periodicidade}

Biblionline

Trimestral, devendo ser publicada nos meses de abril, agosto e dezembro. Recebe submissões em fluxo contínuo.

Encontros Bibli Quadrimestral, mas adotou a modalidade de publicação continuada a partir do volume 24, ano 2019.

\section{Dados abertos}

Biblionline

Não especifica.

Encontros Bibli Permite submissão de artigo de dados.

\section{Composição da equipe editorial}

Biblionline

A equipe editorial é composta por 43 membros:

1 Editor científico

3 Comissão editorial

27 Conselho consultivo local

12 Conselho consultivo externo

Encontros Bibli A equipe editorial é composta por 22 membros:

3 Editores

14 Conselho editorial

1 Revisor de texto

2 Revisor de diagramação e conferência

2 Ficha catalográfica

\section{Critérios para a construção do conselho editorial}

Biblionline

Não menciona

Encontros Bibli

Não menciona

Composição do conselho editorial

Biblionline

Conselho consultivo - 39 membros sendo:

15 Sem identificação

BRASIL

18 Universidade Federal da Paraíba 


\begin{tabular}{|c|c|}
\hline & $\begin{array}{l}2 \text { Universidade Federal de São Carlos } \\
1 \text { Universidade de Brasília } \\
1 \text { Universidade Federal de Lavras } \\
1 \text { Universidade Federal do Rio de Janeiro } \\
1 \text { Universidade Federal do Rio Grande do Norte }\end{array}$ \\
\hline Encontros Bibli & $\begin{array}{l}\text { Conselho editorial - } 14 \text { membros sendo: } \\
\text { BRASIL } \\
3 \text { Universidade de São Paulo } \\
1 \text { Universidade Estadual de Londrina } \\
1 \text { Universidade Federal de Minas Gerais } \\
1 \text { Universidade Federal da Paraíba } \\
1 \text { Universidade de Brasília } \\
\text { ESPANHA } \\
1 \text { Université Paul Valéry Monptellier } 3 \\
1 \text { Universidad Carlos III de Madrid } \\
1 \text { Universidad de Granada } \\
1 \text { Universidad Complutense de Madrid } \\
\text { ESTADOS UNIDOS } \\
1 \text { Universidade da Califórnia em Riverside } \\
\text { URUGUAI } \\
1 \text { Universidad de la República } \\
\text { ARGENTINA } \\
1 \text { Universidad Nacional de Cuyo }\end{array}$ \\
\hline \multicolumn{2}{|c|}{ Nível de inserção do periódico no cenário internacional } \\
\hline Biblionline & Não especifica. \\
\hline Encontros Bibli & $\begin{array}{l}\text { Possui índice H de } 28 \text { e o Índice i10 de } 109 \text {, formulado a } \\
\text { partir do Google Acadêmico em fevereiro de } 2019 \text {. }\end{array}$ \\
\hline \multicolumn{2}{|r|}{ Tempo de avaliação } \\
\hline Biblionline & $\begin{array}{l}\text { A média da edição atual é de } 65 \text { dias (v. } 15 \text { n. } 3 \text { (2019). O } \\
\text { artigo publicado com menor tempo foi } 11 \text { dias e o que } \\
\text { demorou mais, foi } 146 \text { dias. }\end{array}$ \\
\hline Encontros Bibli & $\begin{array}{l}\text { O tempo médio de resposta desde a aceitação do original até } \\
\text { a confirmação de publicação é de aproximadamente } 120 \text { dias. }\end{array}$ \\
\hline
\end{tabular}


Biblionline

Apresenta critérios: coesão, coerência e clareza textual; pertinência da temática em relação à área; originalidade; pertinência metodológica; adequação às normas da ABNT. O sistema de submissão adota a revisão duplo cega por pares.

Encontros Bibli Avaliação por Pares Cega. Permite o tipo de relatórios abertos, quando o relatório do parecerista é publicizado.

\section{Rede colaboração da revista}

Biblionline

Composta por 27 membros do Conselho Consultivo local e 12 membros do Conselho Consultivo externo nacional. Sem membro externo, como representado na figura 5.

Figura 5 - Grafo membros conselho consultivo periódico Biblionline

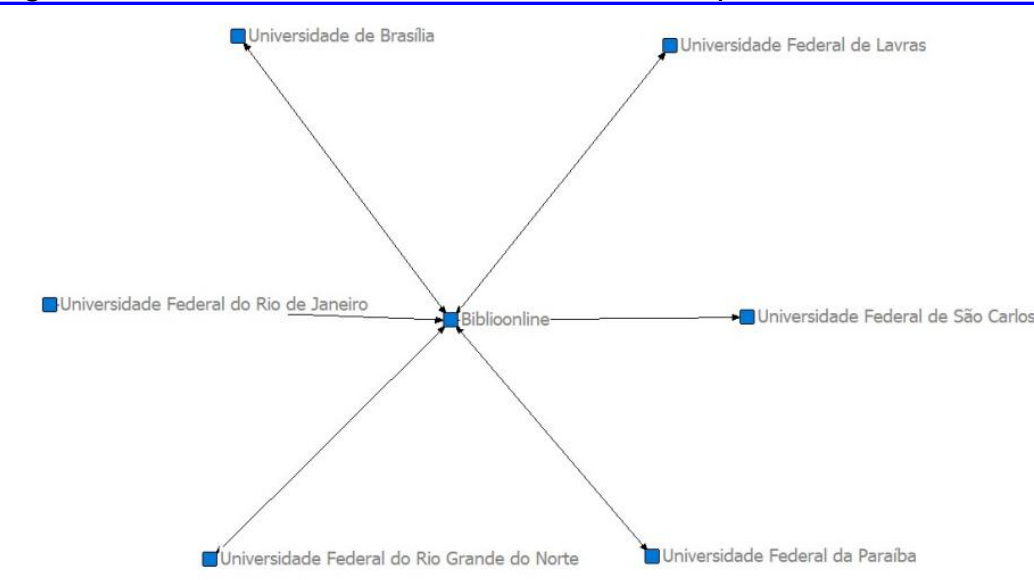

Fonte: Biblionline (2020)

Encontros Bibli Equipe editorial formada por 14 membros, 7 de universidades brasileiras e 7 de universidades estrangeiras, como representado na figura 6 .

Figura 6 - Grafo membros conselho editorial periódico Encontros Bibli 


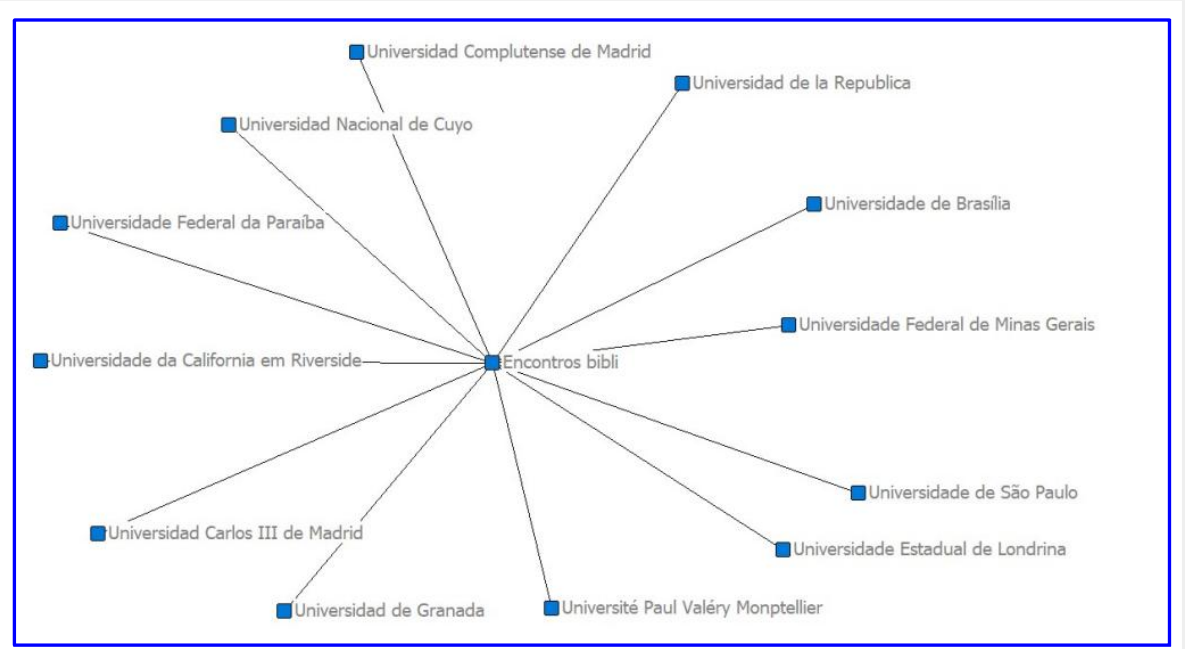

Fonte: Encontros Bibli (2020)

\section{Cobertura temática do artigo}

Biblionline

Encontros Bibli
Biblioteconomia, Arquivologia, Ciência da Informação e Museologia e áreas correlatas.

Ciência da Informação, Biblioteconomia, Arquivologia, Museologia e áreas correlatas.

\section{Diretrizes para autores indexadores da revista}

Biblionline

Encontros Bibli
Não especifica.

\section{Fontes de Indexação}

Bases de dados

Web of Science (Emerging Sources Citation index)

Academic Journals Database

BRAPCI - Base de Dados Referenciais de Artigos de

Periódicos de Ciência da Informação

DOAJ Directory of Open Access Journals

Redalyc - Red de Revistas Científicas de América Latina, el

Caribe, España y Portugal

REDIB: Red Iberoamericana de Información y Conocimiento Científico

Sumarios.org - Sumários de Revistas Brasileiras

vLEX - Base de Dados da Área de Direito

\section{Diretórios}

ISSN - ROAD Directory of Open Access Scholarly Resources LATINDEX - Sistema Regional de Información en Línea para Revistas Científicas de América Latina, el Caribe, España y Portugal 


\begin{tabular}{|l|l|}
\hline $\begin{array}{l}\text { Sherpa/Romeo } \\
\text { ULRICHS - Ulrichs Periodical Directory }\end{array}$ \\
& $\begin{array}{l}\text { Portais } \\
\text { Portal de Periódicos - CAPES } \\
\text { World Wide Science } \\
\text { Repositórios } \\
\text { RCAAP - Repositório Científico de Acesso Aberto de Portugal }\end{array}$ \\
\hline Arquitetura da informação adotada pela revista \\
\hline Biblionline & Utiliza o Open Journal Search (OJS) \\
\hline Encontros Bibli & Utiliza o Open Journal Search (OJS) \\
\hline Biblionline & Sim \\
\hline Encontros Bibli & sim \\
\hline Fonte: & \\
\hline
\end{tabular}

Fonte: Dados da pesquisa.

\section{CONSIDERAÇÕES}

O objetivo do presente estudo foi averiguar similaridades e diferenças em relação à política editorial de dois periódicos da área da Ciência da Informação. Pelas categorias descritas, as principais dissimilitudes observadas se referem a equipe editorial e aos indexadores.

Quanto à equipe editorial, apesar do periódico Biblionline ter maior quantitativo, observa-se que 38\% não estão com o vínculo institucional mencionados e há uma alta endogenia. Além disso, não possui integrante do exterior e a rede de colaboração é a metade em comparação ao Encontros Bibli.

Sobre os indexadores, enquanto a Biblionline não menciona a questão, a Encontros bibli está presente em 8 Bases de dados, 4 Diretórios, 2 Portais e 1 Repositório. Quanto maior for o número de indexadores, maior será a possibilidade 
de estabelecer um nível de qualidade elevado, uma vez que é preciso atender a um número considerável de exigências para fazer parte do acervo, além de oferecer ao pesquisador maior recuperabilidade de seus artigos, e o aumento do índice de citações (RIOS; LUCAS, 2020).

Para que a informação se torne mais acessível para a comunidade científica, com o aumento da produção científica, surge a necessidade de indexar os periódicos em bases de dados, diretórios e portais (RIOS; LUCAS, 2020). São plataformas que fornecem informações de artigos e facilitam a localização do material de interesse sem que seja necessário procurar minuciosamente todos os periódicos da área em questão (UNIVERSIDADE FEDERAL DE SANTA CATARINA, 2015).

Assim, considera-se indícios que esses dois pontos sejam possíveis de impactar nos indicadores dos periódicos. Admite-se que é preciso um estudo mais aprofundado para certificar tal evidência.

\section{REFERÊNCIAS}

BARATA, Rita de Cássia Barradas. Dez coisas que você deveria saber sobre o Qualis. RBPG, v. 13, n. 1, janeiro/abril, 2016. Disponível em: https://goo.gl/uxm6er. Acesso em: 18 ago. 2020.

BARROS, A. J. S.; LEHFELD, N. A. S. Fundamentos de metodologia científica. 3. ed. São Paulo: Pearson Prentice Hall, 2007. 147 p.

BIBLIONLINE. João Pessoa: Universidade Federal da Paraíba, 2005-. ISSN 18094775. Disponível em:https://periodicos.ufpb.br/index.php/biblio. Acesso em: 18 ago. 2020.

CONTREIRA, Ingrid Machado; MIRANDA, Angélica C. D. Critérios de qualidade brasileiros e estrangeiros: análise dos periódicos da psicologia. In: MIRANDA, Angélica C. D.; DAMASIO, Edilson; FIRME, Simone Machado (Org.). Ciência Aberta [recurso eletrônico]: visão e contribuição a partir dos periódicos científicos. Rio Grande, RS: Ed. da FURG, 2020. p. 134-156. Disponível em: http://repositorio.furg.br/bitstream/handle/1/8792/CIENCIA\%20ABERTA.pdf?sequenc e=3. Acesso em 18 ago. 2020.

COSTA, H.; CANTO, F. L. DO; PINTO, A. L. Google Scholar Metrics e a proposta do novo Qualis: impacto dos periódicos brasileiros de Ciência da Informação. Informação \& Sociedade: Estudos, v. 30, n. 1, 31 mar. 2020. Disponível em: https://periodicos.ufpb.br/ojs2/index.php/ies/article/view/50676. Acesso em: 18 ago. 2020. DOI:https://doi.org/10.22478/ufpb.1809-4783.2020v30n1.50676 
COORDENAÇÃO DE APERFEIÇOAMENTO DE PESSOAL DE NÍVEL SUPERIOR. Plataforma sucupira: qualis periódicos. Brasília: Ministério da Educação, 2020. Disponível em:

https://sucupira.capes.gov.br/sucupira/public/consultas/coleta/veiculoPublicacaoQuali s/listaConsultaGeralPeriodicos.jsf. Acesso em: 16 ago. 2020.

ENCONTROS BIBLI. Florianópolis: Universidade Federal de Santa Catarina, 1996-. ISSN 1518-2924. Disponível em: https://periodicos.ufsc.br/index.php/eb/index. Acesso em: 18 ago. 2020.

GIL, A.C. Métodos e técnicas de pesquisa social. 4 ed. São Paulo: Atlas, 1994. 207p.

GODOY, A. A. Uma revisão histórica dos principais autores e obras que refletem esta metodologia de pesquisa em Ciências Sociais. Revista de Administração de Empresas, São Paulo, v. 35, n. 2, p. 57-63 Mar./Abr. 1995. Disponível em:

http://bibliotecadigital.fgv.br/ojs/index.php/rae/article/viewFile/38183/36927. Acesso em: 18 ago. 2020.

GOOGLE SCHOLAR METRICS. 2020. Disponível em: https://scholar.google.com/citations?hl=en\&view_op=search_venues\&vq=encontros+ bibli\&btnG=. Acesso em: 18 ago. 2020.

PINTO, A. C.; ANDRADE, J. B. Fator de impacto de revistas científicas: qual o significado deste parâmetro? Química Nova, São Paulo, v. 22, n. 3, p. 448-453, jun. 1999. Disponível em: http://www.scielo.br/scielo.php?script=sci_arttext\&pid=S010040421999000300026\&lng=en\&nrm=iso. Acesso em: 19 ago. 2020.

PRODANOV, C. C. FREITAS, E. C. Metodologia do trabalho científico: métodos e técnicas da pesquisa e do trabalho acadêmico. 2.. ed. Novo Hamburgo: Feevale, 2013. Disponível em: http://www.feevale.br/Comum/midias/8807f05a-14d0-4d5bb1ad-1538f3aef538/E-book\%20Metodologia\%20do\%20Trabalho\%20Cientifico.pdf. Acesso em: 18 ago. 2020.

RIBEIRO, Maria Piedade Fernandes. A importância da indexação para a difusão do conhecimento comunicado as revistas técnico-científicas. Revista Mineira de Enfermagem, v. 10, n. 1, jan./mar. 2006. Disponível em: http://bit.ly/2WDaZBL. Acesso em: 20 ago. 2020.

RIOS, Fahima Pinto; LUCAS, Elaine R. de Oliveira. Critérios para indexação de periódicos científicos. In: MIRANDA, Angélica C. D.; DAMASIO, Edilson; FIRME, Simone Machado (Org.). Ciência Aberta [recurso eletrônico]: visão e contribuição a partir dos periódicos científicos. Rio Grande, RS: Ed. da FURG, 2020. p. 49-66. Disponível em:

http://repositorio.furg.br/bitstream/handle/1/8792/CIENCIA\%20ABERTA.pdf?sequenc e=3. Acesso em 18 ago. 2020.

UNIVERSIDADE FEDERAL DE SANTA CATARINA. Laboratório de Periódicos Científicos. Indexadores para periódicos científicos: critérios de avaliação. Florianópolis: UFSC, 2015. Disponível em: https://bit.ly/2QoIRP8. Acesso em: 18 ago. 2020. 
ZHANG, Y.; LUN, H. Is Google Scholar useful for the evaluation of non-English scientific journals? The case of Chinese journals. Learned Publishing, v. 32, p. 154162, 2019. DOI 10.1002/leap.1208.

\begin{abstract}
Disseminating new knowledge and relevant to the area of expertise should be the priority of a scientific journal. For this, a well-structured editorial policy is necessary to guarantee its quality and sustainability. In this context, the objective of this study was to analyze the editorial policy of journals in the area of Information Science: Biblionline and Encontros Bibli to investigate similarities and differences in the work guidelines proposed by these titles influence the impact of their publications and their classification in evaluation tools. It is a descriptive research because it proposes the description of the characteristics of the journals and establishes relationships between variables. From the point of view of technical procedures, it is a documentary research, since it uses existing documents that have not received analytical treatment. The analysis bias is qualitative, since it raises data that expose the quality of the journals under analysis. For data collection, the main sources were the website of the Biblionline and Encontros Bibli journals. In addition, an instrument with 10 criteria related to publishing scientific journals was built. As technological inputs, he used a collaborative spreadsheet, a document shared on Google and Ucinet to create graphs. It was considered that endogeny and indexers are indications of possible impacts for the indicators of the journals.
\end{abstract}

Keywords: Scientific Journals. Editorial policies. Quality Indicators. 